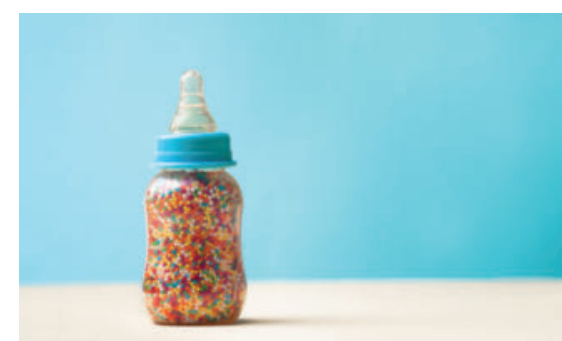

Nach einer Untersuchung der WHO dürfte Babynahrung häufig nicht den Hinweis "geeignet für Babys unter 6 Monaten“ tragen. Vor allem der Zuckergehalt ist bei vielen Produkten zu hoch. (Foto: lithiumphoto - stock.adobe. com, Symbolbild)

Weltgesundheitsorganisation

\section{WHO warnt vor Zucker in Babynahrung}

Seit Langem ist bekannt, dass gesunde Ernährung in der frühen Kindheit vor späteren Erkrankungen wie Bluthochdruck, Diabetes, Herz-Kreislauf-Erkrankungen und Asthma schützen kann. Zwei neue Studien der Weltgesundheitsorganisation (WHO) haben nun ergeben, dass zahlreiche Lebensmittel mit dem Hinweis „geeignet für Babys unter 6 Monaten“ falsch deklariert sind und häufig zu viel Zucker enthalten. In vier europäischen Großstädten (Wien, Sofia, Budapest, Haifa) testete die WHO zwischen November 2017 und Januar 2018 rund 8000 Produkte aus mehr als 500 Geschäften. Davon wurden 28$60 \%$ als geeignet für Babys unter 6 Monaten ausgewiesen. In mehr als der Hälfte der untersuchten Lebensmittel stammten rund $30 \%$ der Kalorien aus Zucker. Auch wenn damit nicht gegen das EU-Recht verstoßen wurde, kritisiert die WHO, dass der Internationale Kodex zur Vermarktung von Muttermilchersatzprodukten nicht eingehalten wurde.

Um dies in Zukunft zu ändern, hat die WHO ein sogenanntes Nutrient Profile Model (NPM) für Kinder im Alter zwischen 6 und 36 Monaten entwickelt. Mit dem NPM sollen Lebensmittel herausgefiltert werden, die nicht als geeignet für Kleinkinder ausgewiesen sein dürften. Das Modell wird nun den Mitgliedsstaaten zur Prüfung und Diskussion vorgelegt.
Quelle: http: / /www.euro.who.int/en/med ia-centre/sections/press-releases/2019/wh oeurope-studies-find-baby-foods-are-hig h-in-sugar-and-inappropriately-marketedfor-babies

Beobachtungs-Kohortenstudie

\section{Wie sicher ist Paracetamol für Schwangere?}

Bei Beschwerden wie Schmerzen, die das Wohlbefinden der Frau stark beeinflussen, ist häufig auch in der Schwangerschaft eine medikamentöse Behandlung notwendig. In diesen Fällen sollte eine sehr sorgfältige Beratung stattfinden, um das Risiko für Mutter und Kind abzuwägen. Schmerzsenkende Wirkstoffe wie Acetylsalicylsäure, Paracetamol, Diclofenac und Ibuprofen gelten in den ersten beiden Schwangerschaftsdritteln als weitgehend unbedenklich. Im dritten Trimester wird nur noch die Einnahme von Paracetamol empfohlen, da die anderen Wirkstoffe beim Fetus einen vorzeitigen Verschluss des Ductus arteriosus Botalli verursachen können.

Forscher von der Berliner Charité haben nun untersucht, inwieweit auch Paracetamol das Risiko für fetale Herz- und Nierenschäden erhöht. Dazu analysierten die Forscher rund 1800 Studien des Instituts für Klinische Pharmakologie und Toxikologie (www.embryotox.de) an der Charité. In der Studiengruppe hatte etwa ein Drittel Paracetamol auch oder ausschließlich im dritten Trimester genommen. In der Kontrollgruppe nahmen zwei Drittel das Medikament ausschließlich im ersten oder zweiten Trimester ein. 60 \% der Schwangeren wandten Paracetamol dabei kürzer als sieben Tage an. Die Forscher verglichen die Studien- und Kontrollgruppe in Bezug auf embryotoxische Ereignisse: Alle dokumentierten Komplikationen standen entweder nicht in Zusammenhang mit der Paracetamoleinnahme oder waren nicht signifikant. Daher wird das Medikament während der gesamten Schwangerschaft als relativ sicher eingestuft.

Quelle: Dathe K et al. Negligible risk of prenatal ductus arteriosus closure or fetal renal impairment after third-trimester paracetamol use: evaluation of the German Embryotox cohort. BJOG 2019; https: / /doi.org/10.1111/1471-0528.15872

Fort- und Weiterbildung

\section{Anpassungslehrgang für zugewanderte Hebammen}

Im Oktober dieses Jahres startet an der Evangelischen Hochschule Berlin (EHB) der erste Anpassungslehrgang für ausländische, zugewanderte Hebammen. Dieser ermöglicht es den Teilnehmenden ihren im Ausland erworbenen Abschluss als Hebamme in Deutschland anerkennen zu lassen. Die Qualifizierungsmaßnahme dauert 12 Monate und umfasst 720 Stunden theoretischen Unterricht, inklusive Sprachtraining sowie 960 Stunden praktische Lernphasen in der Klinik und in der außerklinischen Hebammenarbeit.

In Kooperation mit den Berliner Hebammenschulen wird der Lehrgang so gestaltet, dass die Teilnehmenden auf vorhandenem Wissen aufbauen und noch fehlende Kompetenzen erlernen können. Die theoretischen Lehrgangsphasen finden an der EHB statt. Hier werden die Teilnehmenden mit modernen Lernformen und -medien auf die Hebammenarbeit im deutschen Gesundheitssystem vorbereitet. Während der praktischen Einsätze in Berliner Geburtskliniken, bei freiberuflich tätigen Hebammen und in hebammengeleiteten Einrichtungen ist die regelmäßige Praxisanleitung der Teilnehmenden vorgesehen.

Finanziert wird die Qualifizierung durch das Förderprogramm „Integration durch Qualifizierung (IQ)“ und ist dadurch für die Teilnehmenden kostenlos. Zugangsvoraussetzungen sind $u$.a. der Abschluss als Hebamme, die gesundheitliche Eignung und ein Sprachzertifikat für Deutsch Niveau B1.

Quelle: https: / /www.eh-berlin.de/third-m ission/zentrale-fort-und-weiterbildungsein richtung/anpassungslehrgang-fuer-zuge wanderte-hebammen.html 
Gefördertes Projekt

\section{Radfahren mit Baby}

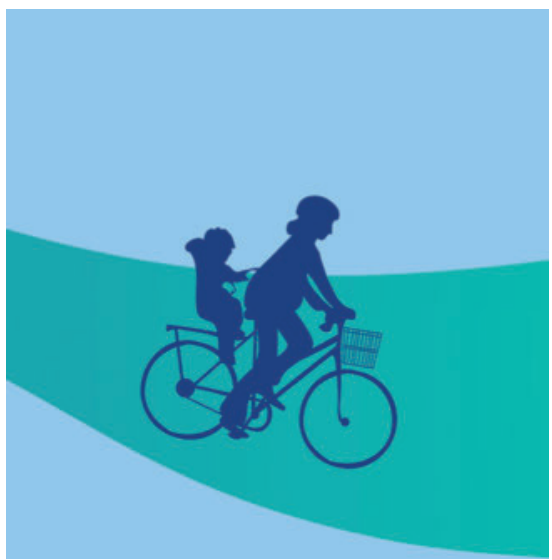

Radfahren mit Baby - sind Schwangere und junge Eltern gut geschützt und informiert, ist Radfahren eine sichere Möglichkeit, sich mit Kind fortzubewegen. (Illustration: Verkehr mit Köpfchen)

Viele Frauen möchten auch in der Schwangerschaft körperlich aktiv sein. Doch gerade beim ersten Kind wissen Schwangere oft nicht, welche Sportarten sie bedenkenlos ausüben können. Sanfte Bewegungsformen wie Schwangerschaftsyoga und Aquafitness sind daher besonders beliebt in der Schwangerschaft. Bei alltäglichen Fortbewegungsmöglichkeiten wie Radfahren sind viele Frauen dagegen vorsichtiger: „Wie sicher ist es, mit wachsendem Bauch zu radeln?"

Deshalb haben sich die Geografin Hannah Eberhardt und die Verkehrsplanerin Anna Gering vom Heidelberger Planungsbüro „Verkehr mit Köpfchen“ diesem Thema gewidmet. In einem dreijährigen vom Bundesministerium für Verkehr und digitale Infrastruktur (BMVI) geförderten Projekt ist die Webseite radfahren-mit-baby. de entstanden. Hier haben die Initiatorinnen Informationen für Schwangere und junge Eltern zusammengetragen. Sie erfahren hier, wie Schwangere ihr Fahrrad für die veränderte Körperhaltung umbauen können und worauf beim Radfahren in der Schwangerschaft zu achten ist. Darüber hinaus informiert die Webseite zu den Themen Radfahren mit Baby, Fahrradanhänger oder Kindersitz.
Wenn Hebammen Eltern bei Fragen zu diesem Thema unterstützen möchten, können sie auf der Homepage ein umfangreiches Handbuch herunterladen, das ihnen Informationen für die Kurs- und Beratungsarbeit liefert. Auch für Eltern gibt es eine Broschüre mit den wichtigsten Fakten. Das Planungsbüro bietet außerdem Schulungen für Hebammen, Workshops für Eltern und Aktionstage an.

Quelle: www.radfahren-mit-baby.de

Retrospektive Studie

\section{Sphinkterverletzungen bei Wassergeburten}

Geburten im Wasser erfreuen sich seit einigen Jahren zunehmender Beliebtheit. Eine retrospektive Studie des Forscherteams um die Gynäkologin Helen Louise Preston am Liverpooler Women's Hospital untersuchte nun mögliche Risiken für die Frauen bei Wassergeburten. Dazu sichteten die Wissenschaftler Dokumentationen von 15734 Geburten, die zwischen 2008 und 2014 in der hebammengeleiteten Geburtsstation stattgefunden hatten. Risikoschwangere waren ebenso von der Analyse ausgeschlossen wie Frauen, bei denen die Geburt eingeleitet oder deren Kind zu früh geboren wurde.

Für eine Wassergeburt entschieden sich 1244 Frauen. Sowohl in der Wassergeburts- als auch in der Kontrollgruppe waren die Frauen ähnlich alt und hatten ein vergleichbares Gewicht. Die Geburtsdauer unterschied sich nicht signifikant. Allerdings benötigten Frauen in der Gebärwanne weitaus weniger Schmerzmittel als die Frauen in der Vergleichsgruppe (12\% vs. $45 \%$ ). Das Risiko für Verletzungen des analen Sphinkters (OASI=obstetric anal sphincter injury) betrug bei allen untersuchten Geburten 1,76\%. Allerdings war die OASI-Rate bei Frauen, die sich für eine Wassergeburt entschieden hatten, deutlich höher als bei Frauen der Vergleichsgruppe (3,3\% vs. $1,6 \%)$. Nach der vorliegenden Studie ist eine Wassergeburt ein unabhängiger Risikofaktor für Verletzungen des analen Sphinkters während der Geburt.
Allerdings kann über die Ursachen bislang nur spekuliert werden. Die Wissenschaftler erklären das erhöhte Risiko mit den in der Badewanne deutlich eingeschränkten Möglichkeiten des Dammschutzes und mit der verstärkten Ödematisierung des perinealen Gewebes.

Quelle: Preston H L et al. Does water birth affect the risk of obstetric anal sphincter injury? Development of a prognostic model. Int Urogynecol J 2019; 30:909-915; doi: https: //doi.org/10.100 7/s00192-019-03879-z

\section{Projekt angelaufen}

\section{Neue Hebammenzentrale in der Region Hannover}

Für viele Schwangere in Deutschland ist es aufgrund des Hebammenmangels schwierig, eine Hebamme zu finden. Um das Problem zu lösen, gibt es in den Regionen Deutschlands verschiedene Ansätze. Die Region Hannover hat nun gemeinsam mit pro familia Niedersachsen e. V. und dem Hebammenverband Niedersachsen e.V. eine Hebammenzentrale gestartet, die die Versorgung von Schwangeren und jungen Familien verbessern soll. Die Zentrale für die Region Hannover bündelt und vermittelt Hebammenhilfen. Zudem berät sie Neu- und Wiedereinsteigerinnen zum Thema Freiberuflichkeit. Drei Hebammen teilen sich die täglichen sechs Stunden Telefonsprechzeit. Die Webseite der Hebammenzentrale soll als Börse fungieren, auf der sich alle freiberuflichen Hebammen aus der Region mit ihren freien Kapazitäten registrieren können.

Das dreijährige Projekt soll durch die Evaluation Daten über die aktuelle Versorgungssituation in der Region Hannover liefern. Die Kosten von 360000 Euro trägt die Region. Die konzeptionelle Begleitung übernimmt der Hebammenverband Niedersachsen, der schon einige Hebammenzentralen in Niedersachsen unterstützt, während die Trägerschaft durch pro familia Niedersachsen erfolgt.

Quelle: www.hebammenzentrale-regionhannover.de. 